\title{
Avaliação da lista de alimentos e porções alimentares de Questionário Quantitativo de Freqüência Alimentar em população adulta
}

\author{
Assessment of the food list and serving \\ size of a Food Frequency Questionnaire \\ in an adult population
}

Luciana Yuki Tomita 1,2

Marly Augusto Cardoso 2

\footnotetext{
1 Núcleo de Investigação em Nutrição, Instituto de Saúde, Secretaria de Estado da Saúde de São Paulo. Rua Santo Amaro 590, São Paulo, SP 01314-000, Brasil. 2 Departamento de Nutrição, Faculdade de Saúde Pública, Universidade de São Paulo. Av. Dr. Arnaldo 715, São Paulo, SP 01246-904, Brasil. marlyac@hotmail.com
}

\begin{abstract}
This study examined both the food list and portion size values from a Food Frequency Questionnaire (FFQ) among participants in a case-control study in São Paulo, Brazil. Two hundred adult respondents to a case-control study were randomly selected (101 men and 99 women; age range: 35-69 years). FFQ food items were examined on the basis of their contribution to total population nutrient intake estimated by 24-hour dietary recall (1-DR). We examined the frequency distribution of equivalent-gram weights of each selected food from the 1-DR and identified the portion sizes as small, medium, and large in the distribution (percentiles 25, 50, and 75, respectively). The FFQ provided close estimation of total fat, saturated fatty acids, cholesterol, total fiber, vitamin B6, and folic acid compared to the 1-DR (paired $t$-test; $p<0.05$ ). The FFQ food list covered the study population's main dietary nutrient sources.
\end{abstract}

Key words Nutritional Surveys; Food Consumption; Food Frequency Questionnaires

Resumo O presente estudo avaliou a adequação da lista de alimentos e das porções alimentares de Questionário Quantitativo de Freqüencia Alimentar (QQFA), entre os participantes de um estudo de casos e controles na cidade de São Paulo, Brasil. O QQFA foi aplicado juntamente com um inquérito recordatório de consumo alimentar de 24 horas (IR24), em amostra aleatória de 200 indivíduos (101 homens, 99 mulheres; idade entre 35 e 69 anos). O tamanho das porções alimentares do QQFA foi classificado em pequeno $(P)$, médio $(M)$ e grande $(G)$ de acordo com distribuição porcentual dos pesos correspondentes às medidas caseiras referidas no IR24 (percentis 25, 50 e 75, respectivamente). A lista de alimentos do QQFA foi analisada segundo a contribuição porcentual de cada item alimentar em relação ao consumo total de nutrientes estimado pelo IR24. Não houve diferença estatisticamente significante entre as médias de consumo estimadas pelo QQFA e pelo IR24 para gordura total, ácidos graxos saturados, colesterol, fibra total, vitamina B6 e folato (teste t pareado; $p<0,05$ ). A lista de alimentos do QQFA mostrou-se adequada à identificação de fontes alimentares na população de estudo.

Palavras-chave Inquéritos Nutricionais; Consumo de Alimentos; Questionários 


\section{Introdução}

Estudos epidemiológicos têm fornecido evidências populacionais sobre a importância da dieta na identificação dos fatores de risco para doenças cardiovasculares, cerebrovasculares, diabetes mellitus e vários tipos de neoplasias malignas. Vários alimentos e nutrientes têm sido relacionados à ocorrência ou prevenção de doenças crônicas em diferentes populações.

Entre os métodos de estimativa de consumo alimentar, a técnica de inquérito recordatório de 24 horas (IR24) é de fácil administração e adequada à descrição de médias ou percentis de consumo alimentar de grupos de indivíduos (Thompson \& Byers, 1994). No entanto, em pesquisa epidemiológica são necessários instrumentos de avaliação de consumo alimentar de fácil aplicação, baixo custo e capazes de caracterizar a dieta habitual dos indivíduos (Block et al., 1986; Thompson, 1994; Willett, 1998). Para essas situações, questionários de freqüência alimentar (QFA) têm sido utilizados em inúmeras investigações. Em estudos analíticos, a magnitude do erro de medida desses instrumentos tem sido avaliada através de estudos de validação e reprodutibilidade (Willett, 1998). No entanto, o desempenho de um QFA validado pode não ser satisfatório ao longo de um mesmo estudo, ou em populações com características diferentes da utilizada em seu estudo de validação.

Os estudos de calibração são particularmente úteis na correção do erro de medida de instrumentos já validados, e têm sido indicados como procedimento alternativo em situações que inviabilizam a realização de inquérito alimentar de vários dias para um novo estudo de validação (Willett, 1998). Este tipo de avaliação tem sido realizada em coortes internacionais (como o EPIC - European Prospective Investigation into Cancer and Nutrition), para a comparação entre as médias de consumo alimentar obtidas no questionário de freqüência alimentar e um único inquérito recordatório de 24 horas (IR24), em amostra representativa da população de estudo (Slimani et al., 2000).

No Brasil, um questionário quantitativo de freqüência alimentar (QQFA) para avaliação da dieta habitual no período de um ano, foi originalmente desenvolvido e validado para a comunidade nipo-brasileira de São Paulo (Cardoso et al., 2001; Cardoso \& Stocco, 2000). Para se conhecer o desempenho do QQFA em população diferente da utilizada no estudo de validação original, o presente trabalho avaliou a abrangência da lista de alimentos e a adequação do tamanho das porções alimentares do QQFA entre os participantes de um estudo de casos e contro- les sobre hábitos alimentares e pólipos adenomatosos em Hospital Nipo-brasileiro de São Paulo.

\section{Indivíduos e métodos}

\section{População de estudo}

O universo amostral do estudo incluiu indivíduos não internados, de ambos os sexos, com idade entre 35 e 69 anos, que realizaram colonoscopia pela primeira vez junto ao Serviço de Endoscopia do Hospital Santa Cruz, São Paulo, incluindo-se pacientes de diferentes origens étnicas $(50 \%$ do total de entrevistados eram japoneses e seus descendentes). Os casos foram definidos pelos pacientes com um ou mais pólipos adenomatosos com confirmação histológica. Os controles foram constituídos pelos pacientes elegíveis ao estudo que não apresentaram pólipos. Foram excluídos do estudo: (a) indivíduos que informavam situações que pudessem interferir no consumo alimentar habitual (gravidez e lactação, por exemplo); (b) pacientes com história ou portadores de polipose familiar; (c) colite de qualquer tipo (por ex., radiação, infecciosa ou idiopática); (d) processo inflamatório do intestino grosso; (e) diverticulite; (f) colectomia prévia; (g) câncer do intestino grosso; (h) colonoscopia incompleta; (i) história de colonoscopia ou adenoma prévios.

No presente estudo, uma amostra de 200 indivíduos foi selecionada aleatoriamente entre todos os casos e controles, estimando-se a inclusão de pelo menos cem casos de adenomas elegíveis ao estudo principal. Para estudos de validação, medidas independentes de consumo alimentar de cem indivíduos têm sido consideradas razoáveis no cálculo de tamanho amostral a partir da transformação Z de Fisher para coeficientes de correlação (Willett, 1998). Já em estudos de calibração, o tamanho amostral mínimo para ótimo índice de participação seria de pelo menos o dobro do número de casos observados, para se garantir nível mínimo de eficiência relativa do estudo (Kaaks et al., 1995). Com base nessas considerações, acreditamos que o tamanho amostral do presente estudo é adequado para fins de avaliação da lista de alimentos e porções alimentares do QQFA entre os participantes do estudo principal.

A participação foi voluntária com consentimento por escrito mediante carta de apresentação do estudo, assegurando-se a confidencialidade das informações e o retorno dos resultados da análise nutricional aos domicílios dos participantes. 
Questionário Quantitativo

de Freqüência Alimentar

Para avaliação da dieta habitual individual no período de um ano, foi utilizado um QQFA originalmente desenvolvido e validado para a comunidade nipo-brasileira de São Paulo (Cardoso et al., 2001; Cardoso \& Stocco, 2000). Para cada item alimentar do QQFA, os participantes referem a freqüência média habitual de consumo de cada item, a respectiva unidade de tempo (se por dia, por semana, por mês ou por ano) e qual o tamanho da porção individual usual (se pequena, média, grande ou extra grande em relação à porção de referência de cada item do QQ FA), obtendo-se assim, a freqüência de consumo como variável contínua e não categórica. A lista de 120 itens alimentares do QQFA, foi elaborada a partir de um inquérito com registro de consumo alimentar de três dias, em amostra aleatória de japoneses de primeira e segunda gerações de São Paulo ( $n=166$; idade entre 45 e 70 anos) (Cardoso et al., 1997). O tamanho das porções alimentares foi classificado em pequeno, médio, grande e extra-grande de acordo com a distribuição porcentual dos pesos equivalentes às medidas caseiras dos registros alimentares. O questionário inclui também outras questões sobre práticas e preferências alimentares habituais, tais como: tipo de adoçante em bebidas, tipo de gordura utilizada no preparo das refeições, número de refeições por dia, ingestão de gordura visível de carnes, outros alimentos não listados de consumo semanal e uso de suplementos dietéticos.

A Tabela 1 ilustra a estrutura geral adotada para o QQFA. Os resultados da avaliação de reprodutibilidade e validação do QQFA foram muito satisfatórios, obtendo-se coeficientes de correlação de Pearson, ajustados pelas calorias totais entre $0,5-0,8$ e 0,3-0,8, respectivamente (Cardoso et al., 2001).

\section{Inquérito recordatório de 24 horas (IR24)}

Um inquérito recordatório de consumo alimentar de 24 horas (IR24), foi obtido através de entrevistas logo após a aplicação do QQFA, registrando-se todos os alimentos e bebidas, e suas respectivas medidas caseiras, consumidos durante um dia antes da realização da colonoscopia. Quando não era possível realizar o IR24 no local da pesquisa (devido à alteração de hábito alimentar na véspera do exame, por exemplo), a entrevista era realizada posteriormente por telefone, utilizando-se o roteiro de entrevista padronizado (Tomita et al., 1999).

\section{Análise dos dados}

A análise do valor nutricional médio das dietas individuais obtidas no QQFA, foi realizada através do programa de computador Dietsys (versão 4.0) (Block et al., 1994), adaptado às características do QQFA. Para a análise das dietas obtidas no IR24, utilizou-se o programa WFOOD 2.0, desenvolvido pela Universidade da Califórnia em Berkely, Estados Unidos. A conversão das medidas caseiras de alimentos e bebidas referidas no IR24, foi realizada com o auxílio de uma lista de equivalência em gramas e mililitros de alimentos e bebidas, organizada a partir de registros alimentares de três dias (Tomita \& Cardoso, 2000). Informações sobre composição química de alimentos foram compiladas das tabelas oficiais do Brasil (IBGE, 1985), do Japão (Resources Council, Science and Tecnology Agency, 1993) e de publicação técnica sobre composição de alimentos nipo-brasileiros (Tomita \& Cardoso, 2000), além dos dados originais da tabela oficial dos Estados Unidos, contidos nos programas para análise das dietas.

Para examinar as médias de nutrientes obtidos no IR24 e no QQFA, calculou-se as diferenças médias para cada nutriente entre os dois métodos, através de teste t de Student pareado após transformação logarítmica dos dados. Para avaliação da lista de alimentos do QQFA, as principais fontes de nutrientes das dietas (para energia, proteínas, gordura total, fibra total, folato, vitaminas A e C) foram identificadas segundo contribuição porcentual de cada item alimentar em relação ao valor nutricional total estimado pelo IR24 para o grupo de indivíduos estudados (Block et al., 1985a, 1985b). Para adequação das porções alimentares do QQFA, calculou-se a mediana e intervalo interquartil dos pesos equivalentes às medidas caseiras referidas no IR24 para cada item do QQFA. Todos os cálculos foram realizados com auxílio de programa de computador SPSS versão 8.0 (SPSS Incorporation, 1997).

\section{Resultados}

Dentre os participantes do presente estudo, 101 (50,5\%) eram do sexo masculino e 99 (49,5\%) do feminino, com idades médias de 55,7 ( DP = 13,7) e 55,0 anos (DP $=14,6)$, respectivamente. No total, $63 \%$ dos participantes eram de origem étnica japonesa.

Os índices de massa corporal médios entre homens e mulheres foram muito próximos: 24,5 $(\mathrm{DP}=4,2)$ e 24,4 ( $\mathrm{DP}=4,2)$, respectivamente. Entre os homens, a prevalência de fumantes foi 
Parte do Questionário Quantitativo de Freqüência Alimentar (QQFA).

\begin{tabular}{|c|c|c|c|c|c|c|c|c|c|c|c|c|c|c|c|c|c|c|c|c|}
\hline \multirow{2}{*}{$\begin{array}{l}\text { Peixes } \\
\text { Peixe frito } \\
\text { (sardinha, pescada) }\end{array}$} & \multicolumn{11}{|c|}{ Quantas vezes você come } & \multicolumn{4}{|c|}{ Unidade } & \multirow{2}{*}{$\begin{array}{l}\text { Porção média (M) } \\
1 \text { unidade média } \\
\text { ou } 2 \text { pedaços }(80 \mathrm{~g})\end{array}$} & \multicolumn{4}{|c|}{ Sua porção } \\
\hline & $\mathrm{N}$ & $\overbrace{}^{1}$ & 2 & 3 & $\stackrel{4}{\bigcirc}$ & 5 & $\stackrel{6}{\bigcirc}$ & $\begin{array}{l}7 \\
\bigcirc\end{array}$ & 8 & 9 & $\begin{array}{l}10 \\
\bigcirc\end{array}$ & $\mathrm{D}$ & $\mathrm{S}$ & $\mathrm{M}$ & $\mathrm{A}$ & & $P$ & $\mathrm{M}$ & $\mathrm{G}$ & $\mathrm{E}$ \\
\hline $\begin{array}{l}\text { Peixe cozido, assado } \\
\text { ou grelhado }\end{array}$ & $\begin{array}{l}\mathrm{N} \\
\mathrm{O}\end{array}$ & ${ }^{1}$ & $\begin{array}{l}2 \\
\bigcirc\end{array}$ & 3 & $\begin{array}{l}4 \\
0\end{array}$ & $\begin{array}{l}5 \\
\bigcirc\end{array}$ & 6 & $\begin{array}{l}7 \\
0\end{array}$ & $\begin{array}{l}8 \\
\bigcirc\end{array}$ & 9 & $\begin{array}{l}10 \\
\bigcirc\end{array}$ & $\begin{array}{l}D \\
O\end{array}$ & $\mathrm{~S}$ & $\mathrm{M}$ & $\begin{array}{l}\mathrm{A} \\
\mathrm{O}\end{array}$ & $\begin{array}{l}1 \text { filé ou } \\
2 \text { pedaços }(80 \mathrm{~g})\end{array}$ & $\mathrm{P}$ & $\mathrm{M}$ & $\mathrm{G}$ & $\mathrm{E}$ \\
\hline $\begin{array}{l}\text { Sashimi (atum, salmão, } \\
\text { polvo, lula, crus) }\end{array}$ & $\begin{array}{l}\mathrm{N} \\
\mathrm{O}\end{array}$ & 1 & $\begin{array}{l}2 \\
\bigcirc\end{array}$ & 3 & $\begin{array}{l}4 \\
\bigcirc\end{array}$ & $\begin{array}{l}5 \\
\bigcirc\end{array}$ & 6 & $\begin{array}{l}7 \\
\bigcirc\end{array}$ & 8 & 9 & $\begin{array}{l}10 \\
\bigcirc\end{array}$ & $\begin{array}{l}\mathrm{D} \\
\mathrm{O}\end{array}$ & $\mathrm{S}$ & $\mathrm{M}$ & $\begin{array}{l}\mathrm{A} \\
\mathrm{O}\end{array}$ & $\begin{array}{l}5 \text { a } 6 \\
\text { fatias }(90 \mathrm{~g})\end{array}$ & $\begin{array}{l}P \\
O\end{array}$ & $\mathrm{M}$ & $\mathrm{G}$ & $\mathrm{E}$ \\
\hline Atum, sardinha ou bonito & $\mathrm{N}$ & 1 & 2 & 3 & 4 & 5 & 6 & 7 & 8 & 9 & 10 & $\mathrm{D}$ & $\mathrm{S}$ & $\mathrm{M}$ & A & 2 colheres de sopa & $P$ & M & G & $E$ \\
\hline em lata & $\bigcirc$ & $\bigcirc$ & $\bigcirc$ & $\bigcirc$ & $\bigcirc$ & $\bigcirc$ & $\bigcirc$ & $\bigcirc$ & $\bigcirc$ & $\bigcirc$ & $\bigcirc$ & $\bigcirc$ & $\bigcirc$ & $\bigcirc$ & $\bigcirc$ & ou lata $(30 \mathrm{~g})$ & $\bigcirc$ & $\bigcirc$ & $\bigcirc$ & $\bigcirc$ \\
\hline \multirow[t]{2}{*}{ chikuwa, kamaboko } & $\mathrm{N}$ & 1 & 2 & 3 & 4 & 5 & 6 & 7 & 8 & 9 & 10 & $D$ & $\mathrm{~S}$ & $\mathrm{M}$ & A & 4 pedaços (80 g) & $P$ & $M$ & $\mathrm{G}$ & $E$ \\
\hline & $\bigcirc$ & $\bigcirc$ & $\bigcirc$ & $\bigcirc$ & $\bigcirc$ & $\bigcirc$ & $\bigcirc$ & $\bigcirc$ & $\bigcirc$ & $\bigcirc$ & $\bigcirc$ & $\bigcirc$ & $\bigcirc$ & $\bigcirc$ & $\bigcirc$ & & $\bigcirc$ & $\bigcirc$ & $\bigcirc$ & $\bigcirc$ \\
\hline
\end{tabular}

Para cada item alimentar do questionário, o entrevistado informa o número de vezes e a unidade de tempo $(D=$ dia, $S=$ semana,

$\mathrm{M}=$ mês e $\mathrm{A}=$ ano) para estimar a freqüência habitual de consumo. Para estimativa da porção alimentar, a porção habitual individual

é referida como $\mathrm{P}=$ pequena; $\mathrm{M}$ = média; $\mathrm{G}$ = grande; e $\mathrm{E}=$ extra-grande, em relação à porção média de referência (porção mediana

calculada segundo distribuição porcentual do tamanho das porções na população de estudo).

de $21 \%$ e de ex-fumantes de $44 \%$; entre as mulheres, $80 \%$ nunca fizeram uso de tabaco, $10 \%$ eram fumantes e $10 \%$ ex-fumantes.

A Tabela 2 mostra o consumo médio e desvio-padrão de energia e nutrientes (excluindose suplementos) estimados pelo QQFA e pelo IR24. Não houve diferença estatisticamente significante entre as médias de consumo estimadas pelos dois métodos para gordura total, ácidos graxos saturados, colesterol, fibra total, vitamina B6 e folato. Para os demais nutrientes analisados, valores médios maiores foram observados na avaliação da dieta habitual do QQ FA. As diferenças relativas (em termos porcentuais) entre os níveis de consumo médio estimados pelo QQFA e pelo IR24, variaram de 1,3 a $15,6 \%$ para energia e macronutrientes, e de $5,0 \%$ a $49,1 \%$ para micronutrientes.

A adequação da lista de alimentos foi avaliada segundo a contribuição porcentual de alguns nutrientes para cada item do QQFA em relação ao consumo total obtido pelo IR24 (Tabela 3). Para todos os nutrientes considerados nesta análise (energia, proteínas, gordura total, fibra total, folato, vitaminas A e C) a contribuição percentual da lista de alimentos do QQFA variou de $88,2 \%$ (para fibra total) a $98,4 \%$ (para vitamina A) do consumo total estimado pelo IR24 para o grupo de indivíduos estudados. Os dez alimentos que mais contribuíram para o consumo total de energia foram: arroz refinado, preparado com óleo $(10,4 \%)$; pão francês, de forma ou italiano $(7,1 \%)$; carne bovina $(6,5 \%)$; massas
$(9,1 \%)$; arroz japonês preparado sem óleo $(6,0 \%)$; carne de frango $(4,5 \%)$, leite integral $(4,1 \%)$; feijão roxo/carioca $(3,2 \%)$; peixes $(3,2 \%)$; bolos, tortas, pavês ou bombas (2,7\%). Juntos, esses 10 itens do QQFA representaram 56,8 \% do total de energia consumido pelo grupo de indivíduos estudados.

A Tabela 4 mostra a distribuição em percentis dos pesos das porções dos alimentos incluídos no QQFA. Na análise da distribuição dos pesos das porções dos alimentos segundo sexo, observaram-se valores semelhantes na maioria dos itens, adotando-se então os valores encontrados na distribuição geral da população estudada. Dessa forma, para avaliação da dieta habitual neste grupo de indivíduos, o tamanho das porções alimentares do QQFA pode ser classificado em pequeno, médio e grande de acordo com os pesos correspondentes aos percentis 25,50 e 75 , respectivamente.

\section{Discussão}

A identificação do papel da dieta na epidemiologia de diversas doenças, e o domínio de recursos estatísticos e computacionais, têm estimulado nas últimas décadas o desenvolvimento e aperfeiçoamento de instrumentos de avaliação da dieta habitual, geralmente definida pelo consumo médio usual de alimentos por período de tempo superior a uma semana (Block et al., 1986; Willett, 1998). 
Consumo médio (desvio-padrão) de energia e nutrientes (excluindo suplementos) estimado pelo Questionário Quantitativo de Freqüência Alimentar (QQFA) e por um inquérito recordatório de 24 horas (IR24) em indivíduos atendidos no Serviço de Endoscopia do Hospital Santa Cruz, São Paulo, Brasil ( $=200)$.

\begin{tabular}{|c|c|c|c|c|}
\hline Nutrientes & QQFA & IR24h & $\begin{array}{l}\text { Diferença média } \\
\text { (g) }\end{array}$ & $\begin{array}{c}\text { Diferença relativa } \\
\text { (\%)a }\end{array}$ \\
\hline Energia total (kcal) & $1996,5(661,9)$ & $1746,1(597,8)$ & $250,4^{\star}$ & 12,5 \\
\hline Proteínas (g) & $79,7(27,6)$ & $72,5(40,9)$ & $7,2^{\star}$ & 9,0 \\
\hline Gordura total (g) & $62,0(28,7)$ & $56,9(30,0)$ & 5,1 & 8,2 \\
\hline Ácidos graxos saturados (g) & $18,2(9,3)$ & $19,8(12,7)$ & $-1,6$ & 8,7 \\
\hline Colesterol (mg) & $215,6(127,3)$ & $212,8(238,0)$ & 2,8 & 1,3 \\
\hline Carboidratos (g) & $277,3(92,4)$ & $234,04(92,5)$ & $43,3^{*}$ & 15,6 \\
\hline Fibra total (g) & $17,7(6,8)$ & $18,1(10,4)$ & $-0,4$ & 2,4 \\
\hline Vitamina A (ER) & $1530,2(896,4)$ & $779,2(680,5)$ & $751,0^{*}$ & 49,1 \\
\hline Tiamina (mg) & $1,4(0,4)$ & $0,9(0,4)$ & $0,4^{*}$ & 32,1 \\
\hline Riboflavina (mg) & $1,7(0,8)$ & $1,1(0,5)$ & $0,7^{*}$ & 38,0 \\
\hline Vitamina B6 (mg) & $1,5(0,5)$ & $1,4(0,8)$ & 0,1 & 6,8 \\
\hline Niacina (mg) & $17,4(5,6)$ & $12,8(10,5)$ & $4,6^{*}$ & 26,6 \\
\hline Vitamina C (mg) & $233,4(142,1)$ & $158,4(144,5)$ & $75,0^{\star}$ & 32,1 \\
\hline Vitamina E (a-TE) & $7,1(2,8)$ & $2,7(3,4)$ & $4,4^{*}$ & 62,0 \\
\hline Folato (mg) & $261,3(109,1)$ & $248,3(151,4)$ & 13,0 & 5,0 \\
\hline Cálcio (mg) & $824,7(409,3)$ & $561,8(349,8)$ & $262,7^{*}$ & 31,8 \\
\hline Fósforo (mg) & $1275,3(459,6)$ & $999,6(424,1)$ & $275,6^{*}$ & 21,6 \\
\hline Potássio (mg) & $3046,6(1326,5)$ & $2570,8(1490,2)$ & $475,8^{*}$ & 15,6 \\
\hline Sódio (mg) & $2737,8(1134,4)$ & $1538,6(5046,4)$ & $1199,2^{*}$ & 43,8 \\
\hline Ferro total $(\mathrm{mg})$ & $14,5(5,2)$ & $10,7(7,0)$ & $3,7^{*}$ & 25,9 \\
\hline
\end{tabular}

a Diferença percentual em relação ao consumo estimado pelo QQFA.

* $p<0,05$ (teste $\mathbf{t}$ de Student pareado).

Sabe-se que qualquer método de avaliação dietética apresenta erros inerentes à técnica empregada, e que nenhuma medida da dieta permite estimar a ingestão "verdadeira" de um indivíduo ou grupo de indivíduos (Thompson \& Byers, 1994; Willett, 1998). Para se conhecer o grau de acurácia de um método de avaliação de consumo alimentar, recomenda-se sua comparação com medidas que utilizem outra técnica de avaliação dietética como referência. Em estudos de validação, o objetivo principal é identificar a relação entre a medida observada (em teste) e a real exposição. Já os estudos de calibração são particularmente importantes em estudos multicêntricos, pois estimam o valor da constante que descreve as relações entre o risco ao desfecho de interesse e a alteração no nível de exposição, permitindo assim, correções no erro de medida do instrumento (Willett, 1998).

O uso de inquérito recordatório de consumo alimentar de 24 horas (IR24), permite aos entrevistadores colher um grande número de informações e detalhes do modo de preparo e ingredientes utilizados em receitas caseiras. Esse mé- todo tem como vantagem a rapidez da aplicação, baixo custo e aceitabilidade por parte dos entrevistados, permitindo obter muitas entrevistas em um curto período, além de não possibilitar ao entrevistado modificar os hábitos alimentares (Buzzard et al., 1996; Thompson \& Byers, 1994). Porém, um único IR24 não permite estimar a dieta habitual individual, mas tem se mostrado útil em comparações de médias de consumo de grupos de indivíduos (Willett, 1998).

No presente estudo, um IR24 foi obtido para avaliar a adequação da lista de alimentos e do tamanho das porções de um questionário quantitativo de freqüência alimentar (QQFA), como etapa inicial de um estudo de calibração. A comparação do consumo médio de nutrientes obtidos pelos dois métodos, observou estimativas semelhantes de ingestão para gordura total, ácidos graxos saturados, colesterol, fibra total, vitamina B6 e folato, sugerindo que o questionário utilizado compreende razoavelmente as fontes alimentares desses nutrientes (Willett, 1998). No entanto, as diferenças encontradas 
Contribuição percentual de nutrientes para alimentos do QQFA em relação ao valor nutricional total obtido em um inquérito recordatório de 24 horas $(n=200)$. Serviço de Endoscopia, Hospital Santa Cruz, São Paulo, Brasil, 1999.

\begin{tabular}{|c|c|c|c|c|c|c|c|}
\hline Alimento & Energia & Proteína & $\begin{array}{l}\text { Gordura } \\
\text { Total }\end{array}$ & Fibra & $\begin{array}{l}\text { Vitamina } \\
\text { A }\end{array}$ & $\begin{array}{l}\text { Vitamina } \\
\text { C }\end{array}$ & Folato \\
\hline Misoshiru & 0,9 & 3,2 & 2,0 & 2,0 & * & * & * \\
\hline Macarronada, ravióli & 6,0 & 4,1 & 0,5 & 4,3 & 0 & 0 & 2,1 \\
\hline Outras massas & 3,1 & 1,7 & 4,4 & 4,0 & 0,3 & 4,6 & 2,9 \\
\hline Estrogonofe, kare-raisu, etc. & 1,9 & 2,7 & 3,8 & 0,2 & 1,8 & 0,1 & 0,4 \\
\hline Carne bovina & 6,5 & 16,4 & 12,2 & 0 & 0 & 0 & 2,0 \\
\hline Carne de frango & 4,5 & 9,0 & 8,7 & 0,6 & 6,0 & 0,1 & 0,7 \\
\hline Carne suína & 0,6 & 1,1 & 1,2 & 0 & 0 & 0 & 0,1 \\
\hline Embutidos (lingüiça, salsicha) & 1,4 & 1,8 & 3,6 & 0 & 0 & 0,2 & 0,1 \\
\hline Presunto, mortadela & 0,6 & 0,8 & 1,6 & 0 & 0 & 0 & 0 \\
\hline Peixes & 3,2 & 14,0 & 2,7 & 0 & 6,9 & 0,3 & 2,2 \\
\hline Feijão roxo/carioca & 3,2 & 5,2 & 0,4 & 15,4 & 0 & 0,3 & 22,7 \\
\hline Feijoada & 0,2 & 0,2 & 0,3 & 0,4 & 0 & 0 & 0,6 \\
\hline $\begin{array}{l}\text { Lentilha, grão de bico, } \\
\text { feijão branco, soja em grãos }\end{array}$ & 0,5 & 0,8 & 0,7 & 1,6 & 0 & 0,3 & 2,4 \\
\hline Tofu fresco e yaki-dofu & 0,4 & 0,6 & 0,9 & 0,3 & 0 & 0 & 0,3 \\
\hline Natto e miso & 0,1 & 0,2 & 0,1 & 0,1 & 0 & 0 & 0,1 \\
\hline Leite de soja & 0,1 & 0,2 & 0,1 & 0,1 & 0 & 0 & 0 \\
\hline Omelete, ovo cru/cozido & 1,0 & 1,3 & 2,6 & 0 & 18,7 & 0 & 2,1 \\
\hline Arroz refinado com óleo & 10,4 & 3,8 & 12,5 & 7,0 & 0 & 11,6 & 6,3 \\
\hline Arroz integral & 0,4 & 0,2 & 0 & 0 & 0 & 0 & 0 \\
\hline Arroz japonês (sem óleo) & 6,0 & 2,7 & 0,3 & 1,3 & 0 & 0 & 0 \\
\hline Batata frita & 0,9 & 0,3 & 1,4 & 0,9 & 0 & 0,3 & 0,6 \\
\hline Leite integral & 4,1 & 4,8 & 7,4 & 0 & 32,2 & 0,7 & 2,6 \\
\hline Leite desnatado & 1,0 & 2,1 & 0,5 & 0 & 0,2 & 0,2 & 0,7 \\
\hline Aveia, cereais matinais & 0,4 & 0,4 & 0,2 & 1,0 & 0 & 0 & 0,2 \\
\hline logurtes, produtos lácteos & 0,7 & 1,0 & 1,1 & 0 & 2,5 & 0,2 & 0,5 \\
\hline Queijo fresco, ricota, cottage & 0,5 & 1,0 & 0,9 & 0 & 3,8 & 0 & 0,3 \\
\hline Mussarela, parmesão, provolone & 1,6 & 2,4 & 4,1 & 0 & 11,6 & 0 & 0,5 \\
\hline Alface/escarola/agrião/rúcula & 0,3 & 0,8 & 0,1 & 3,0 & 0 & 2,8 & 9,7 \\
\hline $\begin{array}{l}\text { Acelga, repolho, couve, } \\
\text { espinafre, brócolos, couve-flor }\end{array}$ & 0,2 & 0,4 & 0,1 & 1,9 & 0 & 3,3 & 2,5 \\
\hline Tomate, beterraba, cenoura & 0,4 & 0,3 & 0,1 & 2,0 & 0 & 1,5 & 1,2 \\
\hline Berinjela/chuchu/abobrinha & 0,2 & 0,2 & 0,1 & 0 & 0 & 0,3 & 0,9 \\
\hline $\begin{array}{l}\text { Pepino, pimentão, broto } \\
\text { de bambu, nabo, bardana }\end{array}$ & 0,1 & 0,2 & 0,1 & 0,5 & 0 & 0,2 & 0,5 \\
\hline Óleo ou azeite em saladas & 1,2 & 0 & 4,0 & 0 & 0 & 0 & 0 \\
\hline Maionese, molhos rosê & 0,3 & 0 & 0,9 & 0 & 0,1 & 0 & 0 \\
\hline Laranja, tangerina, mexerica & 1,7 & 0,8 & 0,1 & 8,2 & 0 & 20,7 & 7,4 \\
\hline Banana & 1,1 & 0,3 & 0,2 & 2,8 & 0 & 1,2 & 1,6 \\
\hline Mamão & 0,7 & 0,3 & 0,1 & 2,9 & 0 & 11,5 & 4,5 \\
\hline Caqui ou manga & 0,5 & 0,1 & 0,1 & 1,2 & 0 & 2,9 & 0,7 \\
\hline $\begin{array}{l}\text { Maçã, pêra, melancia, } \\
\text { melão, abacaxi e outras }\end{array}$ & 0,9 & 1,2 & 0,5 & 3,3 & 0 & 2,9 & 0,8 \\
\hline Salada de frutas, frutas em calda & 1,5 & 0,5 & 1,6 & 1,0 & 0,6 & 1,7 & 0,4 \\
\hline Suco natural de laranja & 0,8 & 0,8 & 0,3 & 7,6 & 0 & 16,5 & 1,3 \\
\hline Suco de outras frutas & 0,7 & 0,3 & 0,1 & 3,0 & 0 & 7,1 & 2,7 \\
\hline Açúcar em café/chás & 2,6 & 0 & 0 & 0 & 0 & 0 & 0 \\
\hline Pão francês/forma/outros & 7,1 & 5,5 & 2,4 & 7,0 & 0,1 & 0 & 5,7 \\
\hline
\end{tabular}

(continua) 


\begin{tabular}{|c|c|c|c|c|c|c|c|}
\hline Alimento & Energia & Proteína & $\begin{array}{l}\text { Gordura } \\
\text { Total }\end{array}$ & Fibra & $\begin{array}{l}\text { Vitamina } \\
\mathrm{A}\end{array}$ & $\begin{array}{l}\text { Vitamina } \\
\text { C }\end{array}$ & Folato \\
\hline Pão integral & 0,4 & 0,4 & 0,1 & 1,3 & 0 & 0 & 0,3 \\
\hline Pão doce ou sovado & 0,4 & 0,3 & 0,1 & 0,4 & 0 & 0 & 0,3 \\
\hline Torradas/cream cracker & 2,3 & 1,0 & 2,2 & 1,1 & 0 & 0 & 1,0 \\
\hline Margarina/manteiga & 1,3 & 0 & 4,3 & 0 & 5,0 & 0 & 0 \\
\hline Requeijão normal & 0,2 & 0 & 0,7 & 0 & 2,0 & 0 & 0 \\
\hline Requeijão light & 0,1 & 0,1 & 0,3 & 0 & 0,8 & 0 & 0 \\
\hline Cerveja & 1,1 & 0,2 & 0 & 0,5 & 0 & 0 & 1,2 \\
\hline Pinga, sake, whisky, vodka & 0,7 & 0,1 & 0 & 0 & 0 & 0 & 0 \\
\hline Sorvete & 0,7 & 0,2 & 1,1 & 0 & 2,8 & 0 & 0,1 \\
\hline Chocolate & 0,4 & 0,1 & 0,5 & 0,5 & 0 & 0 & 0 \\
\hline Bolo, tortas, pavês, bombas & 2,7 & 0,9 & 3,1 & 0,5 & 2,7 & 0 & 0,6 \\
\hline Pudins, flans, curau & 0,2 & 0,1 & 0,1 & 0 & 0,3 & 0 & 0 \\
\hline Amendoim, castanha, nozes & 0,2 & 0,2 & 0,5 & 0,3 & 0 & 0 & 0,3 \\
\hline Salgadinho, torresmo & 0,1 & 0,1 & 0,1 & 0 & 0 & 0 & 0 \\
\hline Refrigerantes & 2,5 & 0 & 0 & 0 & 0 & 0 & 0 \\
\hline Sucos artificiais & 0,5 & 0 & 0 & 0 & 0 & 0 & 0 \\
\hline $\begin{array}{l}\text { Total (\% do consumo } \\
\text { populacional) }\end{array}$ & 97,9 & 97,4 & 98,2 & 88,2 & 98,4 & 91,5 & 94,0 \\
\hline
\end{tabular}

* Nutrientes não determinados na análise de composição química do misoshiru.

nessa comparação para a ingestão dos demais nutrientes analisados eram esperadas, uma vez que um único IR24 não considera a variação de consumo alimentar intra-individual, especialmente para micronutrientes cujo consumo na dieta pode variar muito de um dia para outro (Thompson \& Byers, 1994).

Na elaboração da lista de alimentos do QQ FA original, adotou-se como critério de inclusão de alimentos em um mesmo item alimentar, a similaridade do valor nutricional por porção usual e a importância de alguns alimentos na epidemiologia de doenças crônicas (Cardoso et al., 2001). No presente estudo, foi possível avaliar a contribuição percentual dessa lista em relação ao consumo total populacional de alguns nutrientes, estimado pelo IR24. Assim, para os nutrientes considerados na análise (energia total, proteínas, gordura total, fibra total, vitaminas A, C e folato), observou-se que a lista de alimentos compreende mais de $85 \%$ do total consumido pelo grupo de indivíduos estudados. Uma abrangência adequada dos alimentos fontes de energia, tem sido recomendada para se garantir estimativa razoável do consumo de vários outros nutrientes que se correlacionam com a ingestão calórica total da dieta (Block et al., 1986). Dessa forma, a lista de alimentos do QQFA mostrou-se adequada à população do presente estudo, com abrangência semelhante à encontrada em outros questionários utilizados em estudos epidemiológicos internacionais
(Block et al., 1985a, 1985b; Thompson \& Byers, 1994).

A contribuição percentual de um alimento em relação ao consumo total de nutrientes de uma população, reflete tanto seu valor nutritivo como também a freqüência de consumo e sua porção usual. Sem esse tipo de análise, fontes alimentares importantes de um determinado nutriente podem ser omitidas na lista de alimentos de um QQFA, como também, alguns alimentos de menor importância no consumo populacional podem ser desnecessários e, por isso, devem ser omitidos para reduzir o tempo de aplicação do instrumento e evitar superestimativa do consumo alimentar (Block et al., 1986).

Outro aspecto importante da presente avaliação, refere-se à identificação dos pesos correspondentes às porções pequena, média e grande para vários itens do QQFA segundo sua distribuição na população de estudo. Porções alimentares padronizadas representam medidas de conveniência e aproximação que não correspondem às quantidades que habitualmente são consumidas por grupos populacionais diversos. Comparando-se a distribuição em porcentis das porções originais do QQFA em publicação anterior (Cardoso et al., 2001), observou-se valores bem maiores no presente estudo para porção média de massas e grande de suco de laranja natural e doces em geral (importantes fontes de energia e vitamina $\mathrm{C}$ na população estudada). Já os pesos corresponden- 
Tabela 4

Distribuição em percentis $(P)$ do tamanho das porções de alimentos consumidos em um inquérito recordatório de 24 horas. Serviço de Endoscopia do Hospital Santa Cruz, São Paulo, Brasil $(n=200)$.

\begin{tabular}{|c|c|c|c|}
\hline Grupo/Alimento & P25 (g) & P50 (g) & P75 (g) \\
\hline \multicolumn{4}{|l|}{ Sopas } \\
\hline Misoshiru & 177 & 185 & 323 \\
\hline \multicolumn{4}{|l|}{ Massas } \\
\hline Macarronada, ravióli & 170 & 328 & 524 \\
\hline Lasanha ou canelone & 187 & 249 & 257 \\
\hline \multicolumn{4}{|l|}{ Carnes } \\
\hline Estrogonofre, sukiyaki, kare-raisu, bife à milanesa & 87 & 146 & 262 \\
\hline Bife bovino, carne assada & 40 & 110 & 117 \\
\hline Frango & 60 & 100 & 139 \\
\hline Presunto ou mortadela & 15 & 28 & 35 \\
\hline \multicolumn{4}{|l|}{ Peixes } \\
\hline Assados ou fritos & 48 & 71 & 134 \\
\hline \multicolumn{4}{|l|}{ Leguminosas } \\
\hline Feijão roxo, carioca & 50 & 74 & 114 \\
\hline \multicolumn{4}{|l|}{ Ovos } \\
\hline Omelete, cru, cozido, frito & 40 & 48 & 94 \\
\hline \multicolumn{4}{|l|}{ Arroz } \\
\hline Arroz branco temperado com óleo & 81 & 115 & 176 \\
\hline Arroz branco sem óleo & 94 & 132 & 187 \\
\hline \multicolumn{4}{|l|}{ Leite e derivados } \\
\hline Leite integral & 100 & 150 & 200 \\
\hline Leite desnatado & 134 & 150 & 200 \\
\hline Queijo fresco, ricota, cottage & 20 & 25 & 50 \\
\hline Mussarela, parmesão, provolone & 10 & 19 & 34 \\
\hline \multicolumn{4}{|l|}{ Vegetais } \\
\hline Alface, escarola, agrião, rúcula cruas & 27 & 40 & 54 \\
\hline Acelga, repolho & 30 & 45 & 71 \\
\hline Tomate cru & 23 & 36 & 48 \\
\hline Couve, espinafre cozido & 24 & 47 & 70 \\
\hline Cenoura crua ou cozida & 22 & 46 & 54 \\
\hline Berinjela, chuchu, abobrinha & 33 & 50 & 78 \\
\hline Pepino, pimentão & 20 & 40 & 51 \\
\hline \multicolumn{4}{|l|}{ Óleos/gorduras } \\
\hline Óleo, azeite em salada & 1 & 2 & 3 \\
\hline Maionese & 10 & 15 & 15 \\
\hline \multicolumn{4}{|l|}{ Frutas e sucos } \\
\hline Laranja, tangerina, mexerica & 123 & 144 & 186 \\
\hline Banana & 40 & 58 & 83 \\
\hline Mamão & 44 & 75 & 88 \\
\hline Caqui ou manga & 50 & 120 & 180 \\
\hline Salada de frutas, frutas em caldas & 24 & 60 & 85 \\
\hline Suco natural de laranja e outras frutas & 200 & 230 & 300 \\
\hline \multicolumn{4}{|l|}{ Café e chás } \\
\hline Café (infusão ou solúvel) & 50 & 60 & 100 \\
\hline Chá preto ou mate & 150 & 200 & 250 \\
\hline Chá verde, ervas, outros & 200 & 225 & 400 \\
\hline Açúcar adicionado no café/chás & 6 & 11 & 15 \\
\hline \multicolumn{4}{|l|}{ Pães e biscoitos } \\
\hline Pão francês, forma, italiano, sírio & 48 & 50 & 50 \\
\hline Torradas & 14 & 21 & 29 \\
\hline Margarina e manteiga & 5 & 8 & 10 \\
\hline \multicolumn{4}{|l|}{ Bebidas alcóolicas } \\
\hline Cerveja & 300 & 350 & 375 \\
\hline Pinga, sake, whisky, vodka & 56 & 100 & 184 \\
\hline \multicolumn{4}{|l|}{ Doces/sobremesas } \\
\hline Bolos, tortas, pavês, bombas & 50 & 68 & 110 \\
\hline Doces de frutas (abóbora e outros) & 60 & 85 & 88 \\
\hline Refrigerantes & 200 & 250 & 350 \\
\hline
\end{tabular}


tes às porções de arroz japonês, queijos, tomate, maionese, couve e espinafre cozidos, mamão e salada de frutas do presente estudo foram menores que os valores atribuídos na versão original do QQFA. Assim, para cálculo final da dieta habitual obtida no QQFA para os participantes do estudo de casos e controles aqui referido, serão considerados os valores das porções pequena, média e grande apresentados na Tabela 3. A lista de alimentos do QQFA não sofrerá alterações. A partir da presente avaliação e conseqüente correção das porções alimentares do QQFA, a acurácia das estimativas de consumo alimentar em nossa população poderá ser avaliada em novos estudos de validação, sobretudo através de correlação entre consumo de alguns nutrientes e indicadores bioquímicos do estado nutricional.

\section{Conclusões}

A lista de alimentos do QQFA mostrou-se adequada à identificação de fontes alimentares na população de estudo. Para avaliação da dieta habitual no grupo de indivíduos estudados devem ser utilizados os valores das porções $\mathrm{P}, \mathrm{M} \mathrm{e} \mathrm{G}$ dos itens alimentares do QQFA aqui apresentados. Novos estudos de validação do QQFA devem ser continuamente desenvolvidos, incluindo-se investigações sobre correlação entre o consumo de alguns nutrientes e indicadores bioquímicos.

\section{Agradecimentos}

A todos os participantes do presente estudo pela colaboração. Ao Dr. Luís Masúo Maruta, chefe do Serviço de Endoscopia do Hospital Santa Cruz, por permitir e colaborar para a realização do presente trabalho. À nutricionista Adriana Ayumi Kida pela colaboração na realização das entrevistas alimentares. À pesquisadora Maria Mercedes L. Escuder pelo auxílio prestado no processamento dos dados. À Fundação de Amparo à Pesquisa do Estado de São Paulo (FAPESP) pelo auxílio à pesquisa (Processo no97/4878-6) e ao Conselho Nacional de Desenvolvimento Científico e Tecnológico pela concessão de bolsa de produtividade em pesquisa (300167/97-0). L. Y. T. foi bolsista de Inciação Científica da FAPESP durante a realização deste estudo (98/ 03777-4). 


\section{Referências}

BLOCK, G.; COYLE, L. M.; HARTMEN, A. M. \& SCOPPA, S. M., 1994. Revision of dietary analysis software for the Health Habits and History Questionnaire. American Journal of Epidemiology, 139: 1190-6.

BLOCK, G.; DRESSER, C. M.; HARTMAN, A. M. \& CARROLL, M. D., 1985a. Nutrient sources in the American diet: Quantitative data from the NHANES II survey. I. Vitamin and minerals. American Journal of Epidemiology, 122:13-26.

BLOCK, G.; DRESSER, C. M.; HARTMAN, A. M. \& CARROLL, M. D., 1985b. Nutrient sources in the American diet: Quantitative data from the NHANES II survey. II. Macronutrients and fats. American Journal of Epidemiology, 122:27-40.

BLOCK, G.; HARTMAN, A. M.; DRESSES, C. M.; CARROLL, M. D.; GANNON, J. \& GARDNER, L., 1986. A data-based approach to diet questionnaire design and testing. American Journal of Epidemiology, 124:453-469.

BUZZARD, I. M.; FAUCETT, C. L.; JEFFERY, R. W.; McBANE, L.; McGOVERN, P.; BAXTER, J. S.; SHAPIRO, A. C.; BLACKBURN, G. L.; CHLEBOWSKI, R. T.; ELASHOFF, R. M. \&WYNDER, E. L., 1996. Monitoring dietary change in a low-fat diet intervention study: Advantages of using 24-hour dietary recalls vs food records. Journal of the American Dietetic Association, 96:574-579.

CARDOSO, M. A.; HAMADA, G. S.; SOUZA, J. M. P.; TSUGANE, S. \& TOKUDOME, S., 1997. Dietary patterns in Japanese migrants to southeastern Brazil and their descendants. Journal of Epidemiology, 7:198-204.

CARDOSO, M. A.; KIDA, A. A.; TOMITA, L. Y. \& STOCCO, P. R., 2001. Reproducibility and validity of a food frequency questionnaire among women of Japanese ancestry living in Brazil. Nutrition Research, 21:725-733.

CARDOSO, M. A. \& STOCCO, P. R., 2000. Desenvolvimento de um questionário quantitativo de freqüência alimentar em imigrantes japoneses e seus descendentes residentes em São Paulo, Brasil. Cadernos de Saúde Pública, 16:107-114.
IBGE (Fundação Instituto Brasileiro de Geografia e Estatística), 1985. Tabela de Composição de Alimentos. Rio de Janeiro: IBGE.

KAAKS, R.; RIBOLI, E. \& van STAVEREN, W., 1995. Calibration of dietary intake measurements in prospective cohort studies. American Journal of Epidemiology, 142:548-556.

RESOURCES COUNCIL, SCIENCE AND TECHNOLOGY AGENCY, 1993. Standard tables of food composition in Japan. $4^{\text {th }}$ Ed. Tokyo: Women's University of Nutrition Press.

SLIMANI, N.; FERRARI, P.; OCKE, M.; WELCH, A.; BOEING, H.; van LIERE, M.; PALA, V.; AMIANO, P.; LAGIOU, A.; MATTISSON, I.; STRIPP, C.; ENGESET, D.; CHARRONDIERE, R.; BUZZARD, M.; van STAVEREN, W. \& RIBOLI, E., 2000. Standardization of the 24-hour diet recall calibration method used in the european prospective investigation into cancer and nutrition (EPIC): General concepts and preliminary results. European Journal of Clinical Nutrition, 54:900-917.

SPSS INCORPORATION, 1997. SPSS for Windows. Statistical Package for the Social Sciences. Release 8.0. Chicago: SPSS Inc.

TOMITA, L. Y. \& CARDOSO, M. A., 2000. Relação de Medidas Caseiras, Composição Química e Receitas de Alimentos Nipo-Brasileiros. São José do Rio Preto: Faculdade de Medicina de São José do Rio Preto.

TOMITA, L. Y.; KIDA, A. A. \& CARDOSO, M. A., 1999. Técnica de entrevista alimentar por telefone para inquérito recordatório de 24 horas. In: V Congresso Brasileiro da Sociedade Brasileira de Alimentação e Nutrição, Anais, p. 201, São Paulo: Sociedade Brasileira de Alimentação e Nutrição.

THOMPSON, F. E. \& BYERS, T., 1994. Dietary assessment resource manual. Journal of Nutrition, 124: 2245-2317.

WILLETT, W., 1998. Nutritional Epidemiology. 2nd Ed. New York: Oxford University Press.

Recebido em 17 de outubro de 2001

Versão final reapresentada em 26 de março de 2002 Aprovado em 18 de junho de 2002 